\title{
Ligation of arabinogalactan to peptidoglycan in the cell wall of Mycobacterium smegmatis requires concomitant synthesis of the two wall polymers
}

\author{
Ian C. Hancock, ${ }^{1}$ Stephen Carman, ${ }^{1}$ Gurdyal S. Besra, ${ }^{1} \dagger$ \\ Patrick J. Brennan ${ }^{2}$ and Emma Waite ${ }^{1}$
}
1 Department of Microbiology and Immunology, Newcastle University, Newcastle upon Tyne NE2 4HH, UK
2 Department of Microbiology, Colorado State University, Fort Collins, CO 80523, USA

\author{
Author for correspondence: Ian C. Hancock. Tel: +44 191222 7704. Fax: +44 1912227736. \\ e-mail: i.c.hancock@ncl.ac.uk
}

To study the late events of cell wall assembly in Mycobacterium smegmatis, specific in vivo radiolabelling of exponentially growing liquid cultures over periods of less than one cell generation were carried out. $\mathbf{N}$-Acetyl$\left[{ }^{14} \mathrm{C}\right]$ glucosamine was used to label peptidoglycan and $\left[{ }^{14} \mathrm{C}\right]$ glucose to label arabinogalactan and arabinomannan. Over periods of several generations, radioactive cell wall material was turned over as soluble autolysis products into the culture fluid. However, turnover of newly synthesized and labelled cell wall was delayed for about one cell generation, implying inside-to-outside growth of the wall as observed in Bacillus. Little radioactive wall material was released into the culture fluid during the first generation of labelling in growing cultures, but the addition of amoxicillin plus the $\beta$-lactamase inhibitor clavulanic acid, at the minimum inhibitory concentration of amoxicillin, led to the release of radioactive peptidoglycan that could be isolated by gel filtration chromatography and contained nearly $3 \mathrm{~mol}$ alanine per glutamic acid residue, indicating that it was linear, un-crosslinked peptidoglycan that had never been substantially cross-linked to the cell wall due to inhibition of transpeptidation by amoxicillin. This peptidoglycan had no covalently attached arabinogalactan. Radioactive arabinogalactan was synthesized and released from the amoxicillin-treated bacteria without attachment to peptidoglycan. The results indicate that during growth, incorporation of arabinogalactan into the cell wall requires its ligation to newly synthesized peptidoglycan and that the peptidoglycan must be undergoing concomitant cross-linking to the inner surface of the cell wall. Inhibition of peptidoglycan transpeptidation prevents ligation of arabinogalactan to peptidoglycan and its consequent incorporation into the wall.

Keywords: mycobacteria, cell wall synthesis, bacterial cell walls

\section{INTRODUCTION}

The processes of cell wall assembly in mycobacteria are of great intrinsic interest, since the biochemistry of ligation reactions between very large, and in some cases very hydrophobic or insoluble, molecules is still poorly understood. Assembly of the mycobacterial wall offers

\footnotetext{
†Present address: School of Biosciences, The University of Birmingham, Edgbaston, Birmingham B15 2TT, UK
}

several potential targets for antimycobacterial drugs that have so far not been successfully exploited, although progress is being made towards developing agents directed against the synthesis of arabinogalactan, mycolic acids and mycolyl transferases.

The main structural element of the cell walls of mycobacteria is a cross-linked network of peptidoglycan in which some of the muramic acid residues of the glycan chain are covalently substituted at C-6 with a complex polysaccharide of arabinose and galacto- 
furanose. This arabinogalactan is in turn acylated, at polysaccharide chain ends distal to its link to peptidoglycan, with long-chain mycolic acids (Mikusova et al., 1996). Previous in vitro studies have elucidated the main features of the pathway for the biosynthesis of arabinogalactan in Mycobacterium smegmatis (Mikusova et al., 1996, 2000; Xin et al., 1997) and have demonstrated that it is essential for growth (Pan et al., 2001). However, the precise sequence of events in the final assembly of these components into the cell wall remains unclear.

In contrast to the widespread information on peptidoglycan synthesis, there is no useful model in the bacterial world for the synthesis of mycolyl arabinogalactan except for the initial step of linker unit formation, which has analogies to the initiation of teichoic acid synthesis in Gram-positive bacteria. Previous work has elucidated the structure of mycolyl arabinogalactan (Besra et al., 1995 ) and the early stages of its biosynthesis from UDPGalf, a $\mathrm{C}_{50}$-polyisoprenyl phosphate-linked Araf and the usual precursors of long-chain fatty acid synthesis (Mikusova et al., 1996, 2000; Besra et al., 1994). However, there are still substantial gaps in our knowledge of the late stages of synthesis of arabinogalactan and peptidoglycan, the ligation of these two components by the formation of a phosphodiester linkage to muramic acid in the peptidoglycan, and their incorporation into the growing cell wall. Recently, the in vitro biosynthesis of polyisoprenyl phosphate-linked arabinogalactan has been demonstrated, showing that the arabinan moiety of the polymer is built up on the lipid-linked galactan by successive arabinosyl transfers from decaprenyl phosphate-linked arabinose, rather than being synthesized separately with subsequent ligation of the two oligosaccharides, and that this process precedes ligation to the cell wall (Xin et al., 1997).

In the work now reported we have taken advantage of the ability of M. smegmatis to take up radioactive $\mathrm{N}$ acetylglucosamine (GlcNAc) from the culture medium and incorporate it directly and specifically into peptidoglycan, to investigate the consequences of inhibition of peptidoglycan assembly into the cell wall on the incorporation of mycolylarabinogalactan.

\section{METHODS}

Strain and culture conditions. All experiments were carried out with Mycobacterium smegmatis $\mathrm{mc}^{2} 155$. For preparation of permeabilized bacteria, cultures were grown in nutrient broth (Difco). For in vivo radiolabelling experiments GASGAG medium was used, consisting of GAS medium (Takayama et al., 1975) containing 0.02\% Tween 80 to ensure dispersed growth, $1 \mathrm{mM}$ GlcNAc and $5 \mathrm{mM}$ glucose to ensure induction of $n a g A$ and suppression of endogenous GlcNAc synthesis (Hancock \& Poxton, 1988). Under these conditions the mean generation time of the culture was approximately $5 \mathrm{~h}$.

Radioactive in vivo labelling with GIcNAc and glucose. All radioisotopes were obtained from Amersham Pharmacia. Small-scale labelling was carried out in exponentially growing liquid cultures in $25 \mathrm{ml}$ batches of GAS-GAG medium in
$250 \mathrm{ml}$ Erlenmeyer flasks, at $37^{\circ} \mathrm{C}$ in an orbital incubator at 200 r.p.m. The mean generation time was 4.5 h. $370 \mathrm{kBq} \mathrm{N}$ acetyl-D-[1-1 $\left.{ }^{14} \mathrm{C}\right]$ glucosamine $\left(1.9 \mathrm{GBq} \mathrm{mol}^{-1}\right)$ or $370 \mathrm{kBq}$ [U${ }^{14} \mathrm{C}$ ]glucose $\left(8 \cdot 8 \mathrm{GBq} \mathrm{mmol}^{-1}\right)$ was added when the $\mathrm{OD}_{600}$ of the culture was $0 \cdot 5$. The molar concentrations of the added radioactive GlcNAc and glucose were insignificant compared to the concentrations of these sugars in the culture medium. Amoxicillin + lithium clavulanate $\left(36 \mu \mathrm{g} \mathrm{ml}^{-1}+4.5 \mu \mathrm{g} \mathrm{ml}^{-1}\right)$ were added simultaneously when required. These were the lowest concentrations that led in combination to complete inhibition of growth without causing detectable autolysis over the next $4 \mathrm{~h}$. At the appropriate time, the bacteria were collected directly by centrifugation and washed by resuspension and centrifugation in GAS-GAG medium. The culture medium was retained for isolation of extracellular products as described below. In other experiments, particularly where the labelling period was short, the bacteria were collected by filtration on nylon $0.45 \mu \mathrm{m}$ filters $(4.5 \mathrm{~cm}$ diameter) and washed with $50 \mathrm{ml}$ cold GAS-GAG medium. The bacteria were recovered by inverting the filter and drawing through it two $5 \mathrm{ml}$ aliquots of GAS-GAG medium under a weak vacuum, followed by centrifugation, lyophilization and weighing.

The culture fluid from radiolabelled cultures was dialysed for $48 \mathrm{~h}$ against several changes of 51 deionized water using dialysis membrane with a nominal molecular size cut-off of $12.5 \mathrm{kDa}$, then lyophilized. In large scale experiments the culture fluid was immediately frozen, then lyophilized and redissolved in $25 \mathrm{ml}$ deionized water before dialysis as above.

For measurement of the kinetics of radiolabelling with GlcNAc, $3.7 \mathrm{MBq} N$-acetyl-D-[1- $\left.{ }^{14} \mathrm{C}\right]$ glucosamine was added to $500 \mathrm{ml}$ culture in GAS-GAG medium when the culture optical density reached $0 \cdot 1$, and incubation was continued as above. Duplicate $25 \mathrm{ml}$ samples of culture were taken at $2.5 \mathrm{~h}$ intervals and the bacteria were recovered by filtration and washed as described above. The filters were dried and radioactivity measured by liquid scintillation counting. The culture fluid from the samples was heated to $100{ }^{\circ} \mathrm{C}$ for $5 \mathrm{~min}$ to inactivate autolysins, then dialysed overnight against $5 \mathrm{l}$ deionized water, freeze-dried and redissolved in $0 \cdot 2 \mathrm{ml}$ water. The samples were applied in $2 \mathrm{~cm}$-wide bands to a sheet of Whatman $3 \mathrm{MM}$ chromatography paper and chromatographed in ethanol- $0.5 \mathrm{M}$ ammonium acetate $\mathrm{pH} 3.8 \quad(5: 2$, $\mathrm{v} / \mathrm{v}$ ) for $8 \mathrm{~h}$. After drying, the origin regions of the paper were cut out and radioactivity in the immobile macromolecular material was measured by liquid scintillation counting. Samples of undialysed culture fluid were chromatographed in the same way, authentic GlcNAc and chito-oligosaccharides containing up to 4 GlcNAc residues (Sigma-Aldrich) were used as standards and were detected by the silver stain for reducing sugars (Trevelyan et al., 1950); $1 \mathrm{~cm}$ bands along the paper were cut out for measurement of radioactivity as above.

Isolation of cell walls. The radiolabelled bacteria from $25 \mathrm{ml}$ culture were suspended in $1.5 \mathrm{ml}$ water and distributed equally between two RiboLyser Blue tubes (Hybaid) containing mineral beads. Aqueous phenol $(0.75 \mathrm{ml} ; 90 \%, \mathrm{w} / \mathrm{w})$ was added to each tube. The samples were shaken in a RiboLyser (Hybaid) for $60 \mathrm{~s}$ and then maintained at $70{ }^{\circ} \mathrm{C}$ for $1 \mathrm{~h}$. After cooling, the aqueous phase was removed and the phenol phase, with the interfacial material and beads, was diluted with $5 \mathrm{ml}$ methanol and mixed well. As soon as the beads had settled out, the supernatant suspension was removed and centrifuged to recover the insoluble cell wall fraction. This was washed once with methanol and then with water. 
Determination of the specificity of wall labelling with radioactive GICNAC. To determine the specificity of the labelling procedure, bacteria were radiolabelled with GlcNAc for $20 \mathrm{~h}$ and washed with $0.1 \mathrm{M}$ GlcNAc to remove residual radioactive sugar. Cell walls were isolated as described above. They contained between $93 \%$ and $95 \%$ of the radioactivity from the bacteria. The wall fraction was treated with $0 \cdot 1 \mathrm{M}$ $\mathrm{HCl}$ at $100{ }^{\circ} \mathrm{C}$ for $30 \mathrm{~min}$ to release arabinogalactan. This solubilized between 1 and $4 \%$ of the radioactivity, possibly representing the GlcNAc of the linkage disaccharide attaching arabinogalactan to peptidoglycan. The remaining insoluble material was examined by acid hydrolysis followed by cellulose TLC as described below, with authentic GlcNAc and $\mathrm{N}$-acetylmuramic acid standards. This yielded two radioactive products that co-chromatographed with authentic glucosamine and muramic acid. No radioactivity was detectable in the regions of the chromatogram corresponding to hexoses and pentoses. Thus, $\mathrm{N}$-acetyl- $\left[{ }^{14} \mathrm{C}\right]$ glucosamine provides specific in vivo labelling of peptidoglycan and, probably, the GlcNAc residue in the arabinogalactan-linkage unit.

Molecular size fractionation by gel filtration chromatography. The lyophilized extracellular material from $25 \mathrm{ml}$ culture was dissolved in $1 \mathrm{ml}$ water and filtered through a $0 \cdot 45 \mu \mathrm{m}$ nylon filter before application to the chromatography column. Gel filtration chromatography was carried out on Sephacryl S-200HR (Pharmacia). A column $(65 \times 1.6 \mathrm{~cm})$ was equilibrated in $10 \mathrm{mM}$ ammonium acetate, $\mathrm{pH} 5$. Elution was carried out in the same buffer at $1 \mathrm{ml} \mathrm{min}{ }^{-1}$ at $25^{\circ} \mathrm{C}$. Fractions $(1 \mathrm{ml})$ were collected and radioactivity was measured by scintillation spectrometry. The void volume of the column was determined with blue dextran $2000\left(M_{\mathrm{r}}\right.$ about $2 \times 10^{6}$; Amersham Pharmacia). Molecular mass standards of yellow dextran $20\left(M_{\mathrm{r}} 20000\right.$; Amersham Pharmacia $)$, hexa-acetylchitohexaose $\left(M_{\mathrm{r}} 1237\right.$; Sigma Aldrich) and $N$-acetyl-D-[1$\left.{ }^{14} \mathrm{C}\right]$ glucosamine (Amersham Pharmacia) were used for calibration purposes.

Analytical procedures. For analysis of hexose and pentose sugars, samples were hydrolysed at $115^{\circ} \mathrm{C}$ for $2 \mathrm{~h}$ in $2 \mathrm{M}$ trifluoroacetic acid and acid was removed by evaporation under vacuum. For hexosamine analysis, samples were hydrolysed at $100^{\circ} \mathrm{C}$ in $4 \mathrm{M} \mathrm{HCl}$ for $4 \mathrm{~h}$, the acid was removed under vacuum, the residue was dissolved in $0.4 \mathrm{ml}$ water and mixed with $0.05 \mathrm{ml}$ acetic anhydride $(1.5 \%$ by vol. in acetone) at room temperature for $5 \mathrm{~min}$ to $\mathrm{N}$-acetylate aminosugars, and the product was dried under vacuum. It was redissolved in water. Sugars were analysed qualitatively by TLC on cellulose thin layer plates (Merck). They were separated by three developments in formic acid/water/tert-butanol/methylethylketone (15:15:40:30, by vol.) and standards of glucose, galactose, mannose, ribose, arabinose, GlcNAc and $\mathrm{N}$ acetylmuramic acid were detected with aniline phthalate $(0 \cdot 1 \mathrm{M}$ phthalic acid, $0 \cdot 1 \mathrm{M}$ aniline in water-saturated $\mathrm{n}$ butanol). Quantitative analysis was carried out by HPLC (Dionex) on a CarboPac PA1 column in an isocratic gradient of $20 \mathrm{mM} \mathrm{NaOH}$, with pulsed amperometric detection. Measured amounts of GlcNAc and N-acetylmuramic acid were hydrolysed under the same conditions to correct for destruction during hydrolysis.

For amino acid analysis, samples were hydrolysed in $6 \mathrm{M} \mathrm{HCl}$ for $20 \mathrm{~h}$ at $110^{\circ} \mathrm{C}$ and analysed on a Beckman automatic amino acid analyser model 6300.

Radioactive sugars separated by cellulose TLC as described above were detected by autoradiography on Kodak Biomax MR-2 film.

\section{RESULTS}

\section{Kinetics of synthesis and turnover of peptidoglycan in growing $M$. smegmatis}

To obtain information about wall assembly, we investigated the radiolabelling of peptidoglycan in exponentially growing cultures of $M$. smegmatis. Growing bacteria took up and incorporated radiolabelled $\mathrm{N}$ acetylglucosamine (GlcNAc) and, in the presence of glucose, incorporated it specifically into the cell wall. The specificity of labelling was examined in cell walls from bacteria grown for $20 \mathrm{~h}$ with radioactive GlcNAc. Cell walls were isolated as described. They contained between $93 \%$ and $95 \%$ of the radioactivity from the bacteria. The wall fraction was treated with $0 \cdot 1 \mathrm{M} \mathrm{HCl}$ at $100{ }^{\circ} \mathrm{C}$ for $30 \mathrm{~min}$ to release arabinogalactan. This solubilized between 1 and $4 \%$ of the radioactivity, possibly representing the GlcNAc of the linkage disaccharide attaching arabinogalactan to peptidoglycan. Cell wall was also hydrolysed in $4 \mathrm{M} \mathrm{HCl}$, aminosugars were $\mathrm{N}$-acetylated and the radioactive sugars were examined by cellulose TLC with authentic GlcNAc and $\mathrm{N}$-acetylmuramic acid standards. Only two radioactive products were obtained, which co-chromatographed with the standards (for $\mathrm{N}$-acetylmuramic acid, $\left.R_{\text {GlcNAc }}=0 \cdot 4\right)$. Similarly, no radioactivity was detectable in the regions of the chromatogram corresponding to hexoses and pentoses in samples hydrolysed in $2 \mathrm{M}$ trifluoroacetic acid. Thus, $\mathrm{N}$-acetyl- $\left[{ }^{14} \mathrm{C}\right]$ glucosamine provides specific in vivo labelling of peptidoglycan and, probably, the relatively small amount of GlcNAc in the arabinogalactan-linkage unit. Using this in vivo labelling technique it was possible to measure peptidoglycan synthesized in one cell generation in $25 \mathrm{ml}$ bacterial culture.

Radioactivity was incorporated both into the bacterial cell wall and into non-diffusible material in the culture supernatant (Fig. 1). Initially, paper chromatography of undialysed culture fluid was carried out. Up to $5 \mathrm{~h}$ only a trace of radioactivity was detectable other than GlcNAc. After $5 \mathrm{~h}$, increasing amounts of radioactive products with mobilities lower than that of GlcNAc appeared, of which more than $90 \%$ remained at the origin of the chromatograms. However, due to the presence of large amounts of culture medium components in the samples, separation was poor. In subsequent experiments, therefore, samples were dialysed before chromatography. This did not alter the amount of radioactivity at the origins of the chromatograms but the small amounts of mobile products were removed. In the chromatography system used, linear chito-oligosaccharides containing more than three GlcNAc residues remain at the origin.

Although radioactive GlcNAc was incorporated into cell peptidoglycan continuously, very little radiolabel appeared in the culture fluid until approximately $5 \mathrm{~h} \mathrm{(1}$ generation) after the start of labelling (Fig. 1). After this time, during exponential growth radioactive macromolecular material was released into the culture fluid at about $15 \%$ of the rate of incorporation into the bacteria. 


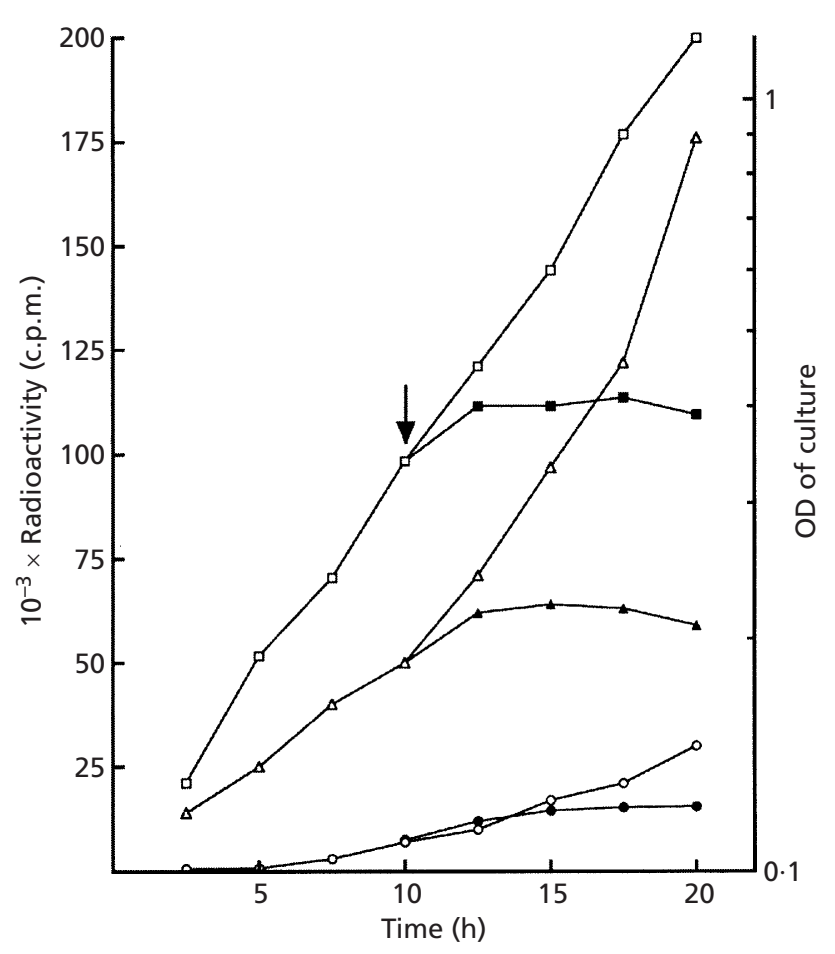

Fig. 1. Kinetics of incorporation and turnover of radioactive GICNAc in a growing culture of $M$. smegmatis. $N$-acetyl-D-[1${ }^{14} \mathrm{C}$ ]glucosamine was added to a $500 \mathrm{ml}$ culture when the culture reached an $\mathrm{OD}_{600}$ of $0 \cdot 1$. Duplicate samples $(25 \mathrm{ml})$ were taken at $2.5 \mathrm{~h}$ intervals and radioactivity in washed bacteria and in macromolecular products in the culture fluid was measured as described in Methods. Amoxicillin and clavulanic acid were added (arrow) $10 \mathrm{~h}$ after addition of the radioactive GICNAc. Figures are the means of duplicate measurements, which differed by less than $8 \% . \square$, Culture optical density; $\triangle$, bacterial counts; $\bigcirc$, counts in medium; $\boldsymbol{\Delta}$, bacterial counts + amoxicillin; $\boldsymbol{O}$, counts in medium + amoxicillin.

Thus, newly synthesized peptidoglycan appeared to be inaccessible to the wall's autolytic systems for about one cell generation. This behaviour is qualitatively similar to that reported for the Gram-positive bacterium Bacillus subtilis where the released GlcNAc-labelled material was shown to be cell wall turnover product (Pooley, 1976). In B. subtilis only old wall material, in the outermost region of the continuously turning-over wall, is susceptible to autolytic enzymes (Pooley, 1976; Merad et al., 1989).

No gross cellular autolysis was caused by amoxicillin as measured by the optical density of the culture or microscopic examination. As shown in Fig. 1, addition of the $\beta$-lactam antibiotic amoxicillin, at its MIC in the presence of the $\beta$-lactamase inhibitor clavulanic acid (Cynamon \& Palmer, 1983), after approximately two cell generations of labelling, inhibited further growth and also blocked incorporation of radioactivity into bacterial cells, but led to a transient increase in release of radioactive peptidoglycan into the culture fluid for about one generation time, relative to the untreated control. Over a longer time period amoxicillin treatment resulted in an overall reduction in extracellular peptidoglycan.

\section{Amoxicillin treatment leads to release of soluble peptidoglycan}

The experiment described above did not indicate whether the transient increase in the release of extracellular peptidoglycan caused by amoxicillin was due to selective autolysis of newly synthesized wall, or to release of new peptidoglycan, due to inhibition of its cross-linking to the wall. To clarify this, we investigated the fate of cell wall components synthesized and specifically radiolabelled during the period equivalent to one cell generation, in the presence and absence of amoxicillin and clavulanic acid added at the same time as the radiolabel. $\mathrm{N}$-Acetyl- $\left[{ }^{14} \mathrm{C}\right]$ glucosamine was used to label peptidoglycan and $\left[{ }^{14} \mathrm{C}\right]$ glucose was used to label arabinogalactan, arabinomannan and other hexose- and pentose-containing polysaccharides (Deng et al., 1995). Glucose labelling was carried out in the presence of GlcNAc to reduce incorporation of label into peptidoglycan.

Cultures were grown to mid-exponential phase and then the antibiotics and the required radiolabelled precursor were added together, and incorporation measured after $4 \mathrm{~h}$. Incorporation of radioactivity into extracellular products in the absence of the antibiotic was very low during this period (less than one cell generation), but amoxicillin caused a ninefold increase in the release of GlcNAc-labelled macromolecular material and a threefold increase in release of glucose-labelled products into the culture fluid, in comparison to the equivalent culture without antibiotic. There was a concomitant $78 \%$ reduction in incorporation from GlcNAc and a $56 \%$ inhibition of incorporation from glucose into the cell wall (in three identical experiments, amounts of incorporated radioactivity differed by $8 \%$ ).

Gel filtration chromatography of the non-diffusible fraction of the culture supernatant revealed the amoxicillin-dependent formation of a new, relatively low molecular mass macromolecular product (Fraction D) labelled with GlcNAc (Fig. 2a) but not glucose (Fig. 2b), indicating that it did not contain arabinogalactan or arabinomannan. In addition there was a small stimulation of labelling of higher molecular mass soluble material of heterogeneous size (Fractions B and C). TLC of the acid hydrolysis products of Fraction D revealed only radioactive glucosamine and muramic acid. Taken in conjunction with the results of the previous experiment showing no overall stimulation of bulk wall turnover or autolysis by amoxicillin, this suggested that amoxicillin specifically activated the autolysis of cell wall synthesized in its presence, or caused the release of new peptidoglycan that was never cross-linked to the wall, as previously observed for B. subtilis and Streptococcus pneumoniae (Waxman et al., 1980; Fischer \& Tomasz, 1984), resulting in the appearance of Fraction D.

To test this hypothesis, we repeated the above experiment on a larger scale and isolated Fractions A-D, equivalent to those shown in Fig. 2, after treatment of the non-diffusible fraction of the culture fluid with 


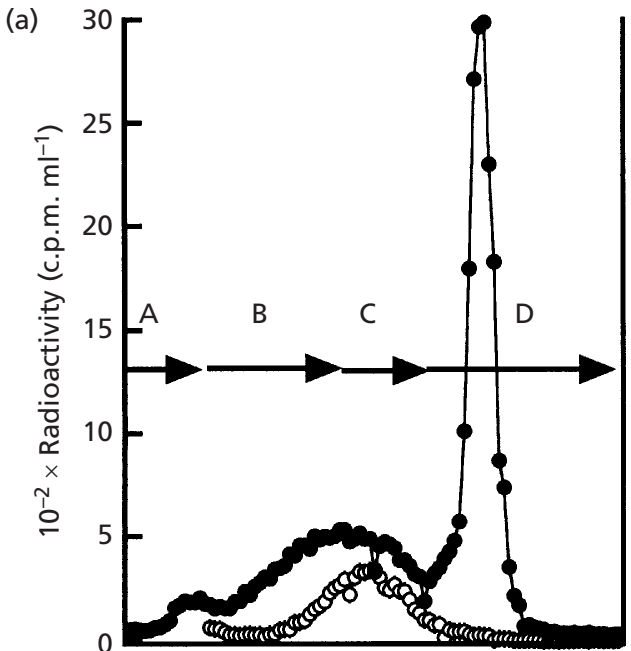

Fraction (b)

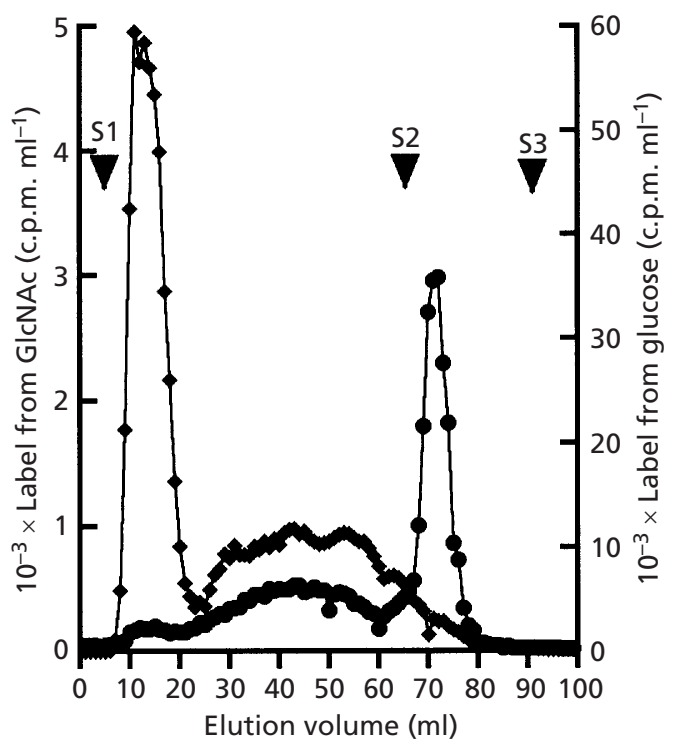

Fig. 2. Gel filtration chromatography of macromolecular extracellular products radiolabelled from exogenous GlcNAc and glucose over $4 \mathrm{~h}$ in an exponentially growing culture. $\mathrm{N}$-acetyl-D-[1-14 C]glucosamine or $\left[\mathrm{U}-{ }^{14} \mathrm{C}\right]$ glucose was added to $25 \mathrm{ml}$ exponentially growing liquid cultures when the $\mathrm{OD}_{600}$ of the culture was 0.5 . Where added, amoxicillin and clavulanic acid were added at the same time. After $4 \mathrm{~h}$, extracellular macromolecular products were isolated and chromatographed on Sephacryl S-200. Fractions $(1 \mathrm{ml})$ were collected and radioactivity measured by liquid scintillation counting. The first fraction shown $(0 \mathrm{ml})$ was collected approximately $5 \mathrm{ml}$ before the void volume had been eluted. (a) Effect of amoxicillin on incorporation from $\mathrm{N}$-acetyl-D-[1-14C]glucosamine. White circles, no amoxicillin; black circles, plus amoxicillin. GICNAc $\left(M_{r}\right.$ 221) eluted $110 \mathrm{ml}$ after the void volume. (b) Comparison of labelling of macromolecular extracellular products by $\mathrm{N}$-acetyl-D-[1-14 C]glucosamine (circles) and $\left[\mathrm{U}-{ }^{14} \mathrm{C}\right]$ glucose (diamonds) in the presence of amoxicillin. The arrowheads indicate the positions of the centres of the elution peaks for the molecular size standards dextran 2000 (S1, mean $\left.M_{\mathrm{r}} 2 \times 10^{6}\right)$, dextran $20\left(\mathrm{~S} 2\right.$, mean $\left.M_{\mathrm{r}} 2 \times 10^{4}\right)$ and hexa-acetylchitohexaose $\left(\mathrm{S} 3, M_{\mathrm{r}} 1237\right)$.

proteinase $\mathrm{K}$ followed by $45 \%$ phenol to remove protein and permit analysis of peptidoglycan amino acids. It was then chromatographed on Sephacryl S200. The protease treatment did not affect the chromatographic profile of the radioactive products. The sugar composition of acid hydrolysates of the fractions were determined by HPLC. In the total extracellular material from the untreated culture ( $3.9 \mathrm{mg}$ hexose), all fractions contained arabinose and mannose, and smaller amounts of galactose, suggesting dispersal of arabinomannans and arabinogalactans of heterogeneous size throughout the chromatogram. Fractions B and C also contained glucosamine and muramic acid, indicating the presence of peptidoglycan. It is important to note that this chemical analysis represented extracellular products accumulated throughout the growth of the culture, not just during the $4 \mathrm{~h}$ radioactive labelling period. From its amino acid composition (see below), the lack of effect of amoxicillin and the presence of arabinose and galactose (Table 1), it seems likely that the peptidoglycan in these fractions was cell wall turnover product. This was not investigated further.

Amoxicillin treatment caused a small increase $(22 \%)$ in the total carbohydrate material in the extracellular products, but caused two very significant changes in the sugar composition of the chromatograph fractions. It led to a large increase in the amounts of arabinose and galactose in Fraction C (1.8- and 6.7-fold, respectively), and to large increases in the amounts of glucosamine and muramic acid (4.3- and 2.8-fold, respectively) in Fraction D. There were smaller increases in arabinose and mannose in Fraction A.

The change in Fraction D corresponded to the formation of the amoxicillin-dependent soluble peptidoglycan identified by radioactive labelling with GlcNAc (Fig. 2). The amino acid and sugar composition of Fraction D (muramic acid/glucosamine/alanine/glutamic acid/diaminopimelic acid in the molar ratio $0 \cdot 9: 1 \cdot 1: 2 \cdot 7: 1 \cdot 1: 1$ ) confirmed that the new amoxicillin-induced product was predominantly peptidoglycan. Fractions B and C also contained peptidoglycan, but its amount was not significantly altered by amoxicillin and it possessed an alanine to muramic acid molar ratio of $1 \cdot 8: 1$, similar to that in the bulk cell wall $(1 \cdot 7: 1)$. In contrast, the Fraction D peptidoglycan exhibited a molar ratio of alanine to muramic acid of $3: 1$, showing that, in contrast to the material in fractions $C$ and $D$, the majority of peptides retained their D-ala-D-ala terminus. This indicated that they had not participated as donors in the peptidoglycan cross-linking transpeptidation reaction. Importantly, there was no concomitant increase in hexoses in this fraction. This observation, the absence of radioactivity derived from glucose in Fraction D (Fig. 2) and the small molecular size of the product (its apparent size was slightly smaller than that of dextran; mean $M_{\mathrm{r}}$ 20000) indicated that the peptidoglycan did not carry covalently linked arabinogalactan. Treatment of the radioactive material with $0 \cdot 2 \mathrm{M} \mathrm{HCl}$ at $100{ }^{\circ} \mathrm{C}$ for 15 
Table 1. Analysis of Sephacryl S-200 chromatography fractions of non-diffusible extracellular products

Fractions A-D were obtained by gel filtration chromatography of non-diffusible extracellular material from cultures with $(+)$ and without $(-)$ treatment with amoxicillin as shown in Fig. 2, were hydrolysed and analysed for sugars by HPLC and for amino acids and aminosugars on an automatic amino acid analyser. ND, Not detected. Hexose and pentose data are the means of three determinations that differed from the mean by less than $10 \%$. Aminosugar data are the means of two determinations. They differed by less than $8.5 \%$. Amino acid composition is given in the text.

\begin{tabular}{|c|c|c|c|c|c|c|c|c|c|c|c|c|c|}
\hline \multirow[t]{3}{*}{ Fraction } & \multirow[t]{3}{*}{ Amox } & \multicolumn{12}{|c|}{ Sugar composition } \\
\hline & & \multicolumn{2}{|c|}{ Ara } & \multicolumn{2}{|c|}{ Gal } & \multicolumn{2}{|c|}{ Man } & \multicolumn{2}{|c|}{ Glc } & \multicolumn{2}{|c|}{ GlcN } & \multicolumn{2}{|c|}{ Mur } \\
\hline & & Amt $†$ & Ratio $\neq$ & Amt $†$ & Ratio $\neq$ & Amt $†$ & Ratio $\neq$ & $\mathrm{Amt} \dagger$ & Ratio $\ddagger$ & $\mathrm{Amt} \dagger$ & Ratio $\neq$ & Amt $†$ & Ratioł \\
\hline \multirow[t]{2}{*}{ A } & - & $4 \cdot 6$ & $0 \cdot 75$ & $1 \cdot 0$ & $0 \cdot 16$ & $6 \cdot 1$ & 1 & $2 \cdot 0$ & $0 \cdot 32$ & ND & 0 & ND & 0 \\
\hline & + & $6 \cdot 7$ & $0 \cdot 73$ & $0 \cdot 85$ & $0 \cdot 09$ & $9 \cdot 1$ & 1 & $2 \cdot 4$ & $0 \cdot 26$ & ND & 0 & ND & 0 \\
\hline \multirow[t]{2}{*}{ B } & - & $5 \cdot 3$ & $2 \cdot 5$ & $0 \cdot 5$ & $0 \cdot 25$ & $2 \cdot 1$ & 1 & ND & 0 & $0 \cdot 5$ & $0 \cdot 24$ & Trace & 0 \\
\hline & + & $6 \cdot 0$ & $2 \cdot 17$ & $0 \cdot 5$ & $0 \cdot 19$ & $2 \cdot 8$ & 1 & $\mathrm{ND}$ & 0 & $0 \cdot 45$ & $0 \cdot 16$ & Trace & 0 \\
\hline \multirow[t]{2}{*}{$\mathrm{C}$} & - & $2 \cdot 5$ & $0 \cdot 49$ & $0 \cdot 8$ & $0 \cdot 15$ & $5 \cdot 1$ & 1 & ND & 0 & $6 \cdot 2$ & $1 \cdot 22$ & $5 \cdot 8$ & $1 \cdot 14$ \\
\hline & + & $4 \cdot 6$ & $0 \cdot 81$ & $5 \cdot 4$ & $0 \cdot 96$ & $5 \cdot 6$ & 1 & ND & 0 & $6 \cdot 1$ & 1.09 & $5 \cdot 4$ & 0.96 \\
\hline \multirow[t]{2}{*}{$\mathrm{D}$} & - & $2 \cdot 6$ & $1 \cdot 24$ & $0 \cdot 5$ & $0 \cdot 17$ & $2 \cdot 1$ & 1 & ND & 0 & $0 \cdot 3$ & $0 \cdot 16$ & $0 \cdot 4$ & $0 \cdot 18$ \\
\hline & + & $2 \cdot 4$ & $1 \cdot 3$ & $0 \cdot 3$ & $0 \cdot 17$ & $1 \cdot 8$ & 1 & $\mathrm{ND}$ & 0 & $1 \cdot 3$ & $0 \cdot 71$ & $1 \cdot 1$ & $0 \cdot 61$ \\
\hline
\end{tabular}

*Amoxicillin treatment.

$\dagger \mu \mathrm{mol}$.

$\ddagger$ Molar ratio; mannose $=1$.

min, conditions that would remove arabinogalactan by hydrolysis of the acid-labile GlcNAc 1-phosphate of the linker unit, had no effect on its chromatographic profile (not shown) also suggesting that the peptidoglycan was not linked to arabinogalactan.

We conclude that the extracellular glucosamine- and muramic acid-containing material formed in the absence of amoxicillin, which only received a small amount of radiolabel during $4 \mathrm{~h}$ growth in the presence of radioactive GlcNAc, represents turnover products of mainly old cell wall, released by the action of autolysins. On the other hand, the radioactive peptidoglycan product in Fraction D, formed over a period of less than one cell generation, and only during treatment with amoxicillin, is linear, un-crosslinked 'nascent' peptidoglycan, or very weakly cross-linked new peptidoglycan that is exceptionally sensitive to autolysin action, released from the bacteria soon after synthesis because transpeptidation was inhibited.

\section{In the absence of normal peptidoglycan cross-linking, arabinogalactan is not ligated to peptidoglycan}

In addition to causing the release of un-crosslinked peptidoglycan in Fraction D, as described above, amoxicillin caused an increase in the amounts of arabinose and galactose in Fraction C. Since there was no concomitant increase in peptidoglycan in these fractions, it was possible that the new material represented arabinogalactan not linked to peptidoglycan. We therefore examined the extracellular products of radioactive glucose labelling over $4 \mathrm{~h}$ in the presence and absence of amoxicillin. Extracellular labelled products were fractionated by gel filtration chromatography as before and Fractions A1D1, equivalent to Fractions A-D of Fig. 2, were collected. During treatment with amoxicillin, incorporation from radioactive glucose into a product eluting from the S-200 column at the void volume (Fraction A1, Fig. 3a) increased approximately $4 \cdot 5$-fold, and into material of smaller, heterogeneous size (Fractions B1 and C1, Fig. 3a) increased twofold, in qualitative agreement with the increases in galactose and arabinose observed by chemical analysis. Total sugar analysis of Fractions A and B (Table 1) revealed principally arabinose and mannose, with a much smaller amount of galactose. The increase in galactose due to amoxicillin treatment was restricted to Fraction C1. Radioactivity incorporated into the sugars from glucose during the $4 \mathrm{~h}$ labelling period in the presence of amoxicillin was examined by TLC of the acid-hydrolysed fractions followed by autoradiography (Fig. 3b). Fraction B synthesized in the presence of amoxicillin contained only radioactive arabinose and mannose in significant amounts, with no detectable galactose, and was therefore predominantly arabinomannan. Fraction A was not fully analysed, but it too contained principally arabinose and mannose, and had a similar radioactive profile (not shown) to Fraction B. It thus appeared that amoxicillin treatment led to release of lipoarabinomannan or of partly degraded arabinan, as previously observed for ethambutol-treated M. smegmatis (Deng et al., 1995).

Fraction C, as shown above, contained mannose, arabinose and galactose in addition to peptidoglycan turnover products. TLC and autoradiography of the hydrolysis products of this fraction (Fig. 3b) showed 

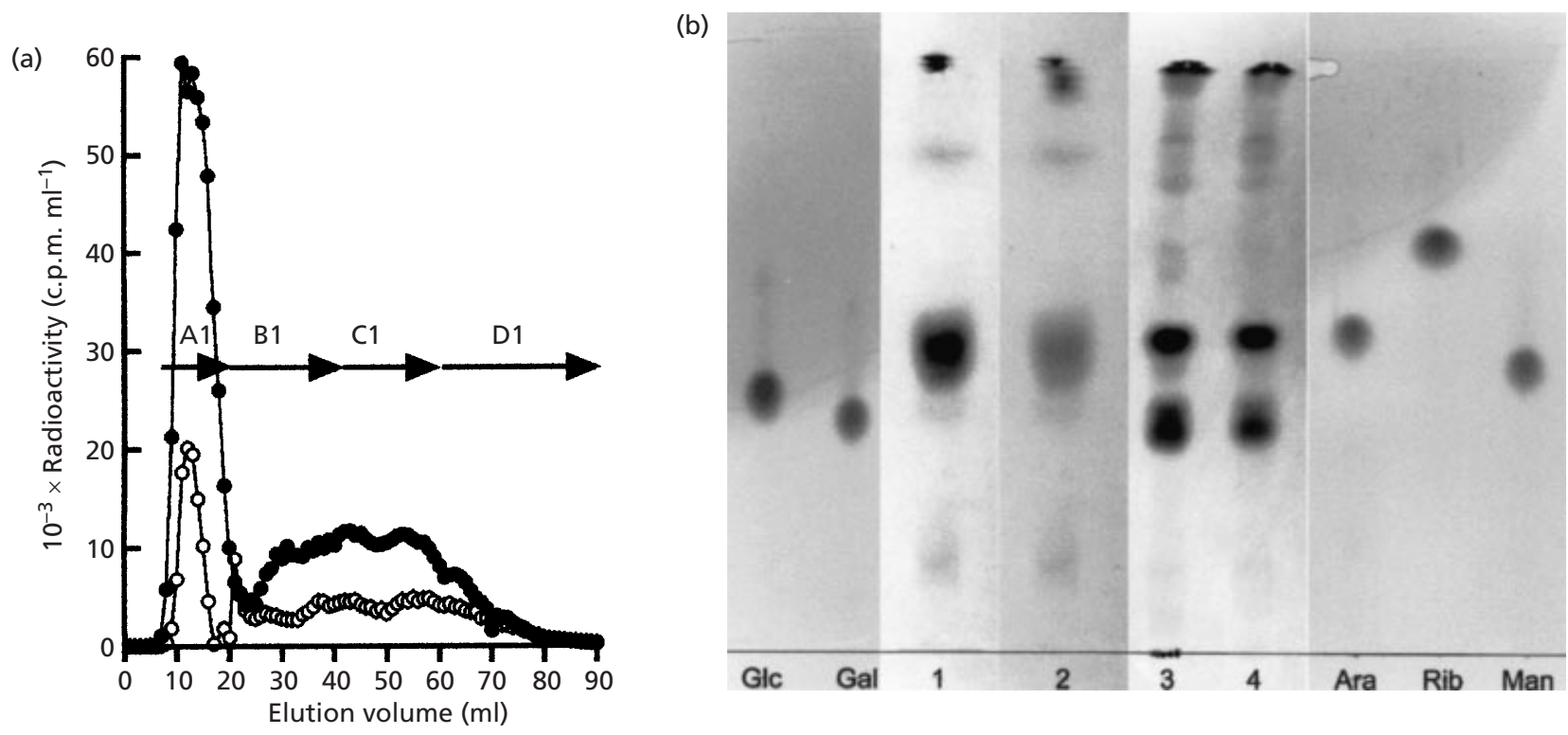

\begin{abstract}
Fig. 3. Effect of amoxicillin on the formation of macromolecular extracellular products radiolabelled from exogenous glucose. (a) Gel filtration of extracellular products: $\left[\mathrm{U}-{ }^{14} \mathrm{C}\right]$ glucose was added to $25 \mathrm{ml}$ exponentially growing liquid cultures when the $\mathrm{OD}_{600}$ of the culture was $0 \cdot 5$. Where used, amoxicillin and clavulanic acid were added at the same time. After $4 \mathrm{~h}$, extracellular macromolecular products were isolated and chromatographed on Sephacryl S-200. Fractions (1 ml) were collected and radioactivity measured by liquid scintillation counting. The first fraction shown $(0 \mathrm{ml})$ was collected approximately $5 \mathrm{ml}$ before the void volume had been eluted. Eluant was pooled into Fractions A1-D1, corresponding to fractions A-D of Fig. 2. White circles, no amoxicillin; black circles, amoxicillin added. (b) TLC of radioactive sugars in acidhydrolysed hexose-containing fractions B1 and C1. Fractions B1 and C1 as shown in Fig. 3a were dialysed, lyophilized and hydrolysed for $2 \mathrm{~h}$ in $2 \mathrm{M}$ trifluoroacetic acid at $115^{\circ} \mathrm{C}$. Cell walls were purified from bacteria radiolabelled with glucose and hydrolysed in the same way. Radioactive sugars in the hydrolysates were separated by cellulose TLC and visualized by autoradiography. Authentic sugar markers were detected with the aniline phthalate reagent. Lane 1, Fraction B1 from amoxicillin-treated culture; lane 2, Fraction C1 with no amoxicillin; lane 3, Fraction C1 from amoxicillin-treated culture; lane 4, cell wall with no amoxicillin. The figure is a composite of autoradiograms of the same TLC plate, exposed for different lengths of time to optimize visualization of each sample.
\end{abstract}

that during the short labelling period radioactive galactose appeared only when the culture was treated with amoxicillin, though arabinose and mannose were labelled both in the presence and absence of the antibiotic (compare lanes 2 and 3). The profile of sugars incorporated into cell wall over the same period in the absence of amoxicillin is shown for comparison (lane 4). Thus, only during amoxicillin treatment the culture synthesized, and released into the medium, a product containing arabinose and galactose (presumably arabinogalactan) that chromatographed in Fraction C1. There was no concomitant increase in glucosamine and muramic acid, indicating that the extra galactose and arabinose were not linked to peptidoglycan.

We conclude that inhibition of peptidoglycan transpeptidation into the cell wall by amoxicillin leads to the separate release into the culture fluid of newly synthesized peptidoglycan, chromatographing in Fraction D, and of arabinogalactan, chromatographing in Fraction $\mathrm{C}$, and that the two polymers are not ligated. Thus when incorporation of newly synthesized peptidoglycan into the cell wall by transpeptidation is blocked, arabinogalactan is not ligated to the peptidoglycan; under normal conditions, new arabinogalactan is ligated to concomitantly synthesized peptidoglycan in a process that requires transpeptidation of the peptidoglycan to the cell wall.

\section{DISCUSSION}

Cell wall assembly in growing bacteria is a process of continuous insertion of new peptidoglycan, with its covalently linked accessory polymers, into the inner surface of the existing wall, where it is attached to older peptidoglycan by transpeptidation. In balanced growth the rate of addition of new material is sufficient to provide for expansion of the growing wall and loss of existing wall by tightly regulated autolysin-catalysed turnover (Merad et al., 1989; Doyle et al., 1988). In Bacillus, the effect of the continual addition of new material at the inner surface of the cell wall and turnover of wall at the outside is that over a period of time, cell wall material migrates from the inner surface to the outer surface where it becomes susceptible to turnover and is released into the culture medium (Pooley, 1976; Archibald et al., 1993). The kinetics of GlcNAc incorporation and turnover shown in Fig. 1 demonstrate a similar process in M. smegmatis. Despite continuous incorporation of radioactive GlcNAc into cellular material, radioactive macromolecular products did not appear in the culture fluid until more than one cell 
generation after addition of radiolabelled GlcNAc to the culture. $\mathrm{N}$-Acetyl- $\left[{ }^{14} \mathrm{C}\right]$ glucosamine would be expected to specifically label peptidoglycan in the presence of unlabelled glucose. The M. tuberculosis genome sequence (Cole et al., 1998) includes genes encoding homologues of the Escherichia coli enzymes NagA, GlmM and GlmU responsible for the uptake of exogenous GlcNAc and its incorporation into UDP-Nacetylglucosamine, the nucleotide precursor of peptidoglycan. In E. coli, NagA is induced by exogenous GlcNAc, which also represses endogenous synthesis of glucosamine 6-phosphate, the normal endogenous precursor of UDP-N-acetylglucosamine. Glucose represses synthesis of glucosamine phosphate deaminase, preventing utilization of exogenous GlcNAc as a carbon and nitrogen source. Thus, in the presence of GlcNAc and glucose, radioactive GlcNAc is incorporated specifically into UDP-N-acetylglucosamine and hence principally into peptidoglycan. This was confirmed by the identification of radioactivity from exogenous GlcNAc only in glucosamine and muramic acid in cell wall hydrolysates.

The delay in appearance of macromolecular radioactive product in the culture fluid (Fig. 1) indicated that peptidoglycan up to at least one cell generation old was not susceptible to turnover. However, when amoxicillin was added at the same time as the radioactive GlcNAc, considerable release of labelled material occurred over the next cell generation and analysis of the product indicated that it consisted of peptidoglycan without covalently attached arabinogalactan. The very high proportion of alanine in this product, approaching $3 \mathrm{~mol}$ $(\text { mol muramic acid })^{-1}$, indicates that a large proportion of the peptide side chains had never participated as donors in a transpeptidation reaction, the process that leads to the incorporation of new peptidoglycan into the cell wall (Cooper et al., 1988; Giles \& Reynolds, 1979; Goodell \& Schwarz, 1983; Ward \& Perkins, 1974), since that process leads to the elimination of one of the three alanines of the nascent pentapeptide. Thus the peptidoglycan was released, either as the result of the action of autolysins on a very abnormally cross-linked subfraction of new cell wall, or without any prior attachment to the cell wall, due in either case to inhibition by amoxicillin of the transpeptidation function but not the chain-extending transglycosylation activity of the high molecular mass penicillin-binding proteins. This peptidoglycan did not carry covalently attached arabinogalactan. We therefore conclude that in vivo, the enzyme(s) catalysing ligation of arabinogalactan specifically act on concomitantly synthesized arabinogalactan and peptidoglycan. Moreover, the peptidoglycan must be in the process of normal transpeptidation to the existing cell wall for ligation of arabinogalactan to occur, since inhibition of transpeptidation by amoxicillin also prevented ligation, though synthesis of neither polymer was inhibited. This suggests that the topology of the complex of ligation enzymes and lipidbound nascent polymers at the cytoplasmic membrane surface is critical for successful ligation.

The ligation of arabinogalactan to peptidoglycan in- volves the transphosphorylation of the terminal $\mathrm{N}$ acetylglucosamine 1-phosphate of the linkage unit of the polymer from its polyisoprenylphosphate carrier to the hydroxyl group at C-6 of an N-glycolylmuramic acid in the peptidoglycan (Mikusova et al., 1996). The reaction closely resembles the ligation of teichoic acid to peptidoglycan in a wide range of Gram-positive bacteria (Archibald et al., 1993) and is an interesting candidate target for new antimicrobial drugs. In the case of teichoic acids, the ligation reaction has been demonstrated in vitro (Hancock, 1981; Ward, 1981) and shown to be insensitive to $\beta$-lactam antibiotics. However, the enzymes and genes responsible for ligation have not been identified in any bacteria.

Similar interdependencies between cross-linking of new peptidoglycan to the cell wall and ligation of accessory wall polymers to the new peptidoglycan have been observed in the cases of wall teichoic acids in B. subtilis (Mauck \& Glaser, 1972) and S. pneumoniae (Fischer \& Tomasz, 1984). The ligation of proteins to peptidoglycan in Gram-positive and Gram-negative bacteria is via penicillin-insensitive transpeptidation reactions that involve sites in the peptidoglycan peptide side chains that would otherwise be available for cross-linking (Navarre \& Schneewind, 1999). Thus protein attachment prevents full cross-linking. The effect of blocking peptidoglycan cross-linking on this process is, however, not known. The phenomenon could have implications for the release of circulating cell wall antigens during the early stages of treatment of bacterial infections with $\beta$ lactam antibiotics (Periti \& Mazzei, 1998). The actions of other antimycobacterial drugs that target specific stages in cell wall synthesis, including ethambutol (Deng et al., 1995) and isoniazid (Bardou et al.,1996), have also been reported to lead to multiple effects on cell wall assembly and stability, including the release of the major surface antigen, lipoarabinomannan.

Progress towards identification of the ordered sequence of late events in the synthesis of mycolylarabinogalactan and its ligation to the cell wall, should be valuable in drug discovery efforts targeting cell wall synthesis, by enhancing our ability to predict the consequences of inhibition of specific targets by new and existing drugs.

\section{ACKNOWLEDGEMENTS}

We thank SmithKline Beecham for the generous gift of clavulanic acid.

This work was supported by grant AI-38087 from the National Cooperative Drug Discovery Groups for the Treatment of Opportunistic Infections (NCDDG-OI), National Institute of Allergy and Infectious Diseases (NIAID) and the National Institutes of Health (NIH), USA.

\section{REFERENCES}

Archibald, A. R., Hancock, I. C. \& Harwood, C. R. (1993). Cell wall structure, synthesis and turnover. In Bacillus subtilis and Other Gram-Positive Bacteria: Biochemistry, Physiology and Molecular Genetics, pp. 381-410. Edited by A. L. Sonenshein, J. A. Hoch and R. Losick. Washington, DC: American Society for Microbiology. 
Bardou, F., Quemard, A., Dupont, M. A., Horn, C., Marchal, G. \& Daffé, M. (1996). Effects of isoniazid on ultrastructure of Mycobacterium aurum and M. tuberculosis and on production of secreted proteins. Antimicrob Agents Chemother 40, 2459-2467.

Besra, G. S., Sievert, T., Lee, R. E., Slayden, R. A., Brennan, P. J. \& Takayama, K. (1994). Identification of the apparent carrier in mycolic acid synthesis. Proc Natl Acad Sci U S A 91, 12735-12739.

Besra, G. S., Khoo, K.-H., McNeil, M. R., Dell, A., Morris, H. R. \& Brennan, P. J. (1995). Interpretation of the structure of the mycolyl-arabinogalactan complex of Mycobacterium tuberculosis as revealed through characterisation of oligoglycosylalditol fragments by fast-atom bombardment mass spectrometry and ${ }^{3} \mathrm{H}$ nuclear resonance spectroscopy. Biochemistry 34, 4257-4266.

Cole, S. T., Brosch, R., Parkhill, J. \& 39 other authors (1998). Deciphering the biology of Mycobacterium tuberculosis from the complete genome sequence. Nature 393, 537-544.

Cooper, S., Hsieh, M.-L. \& Guenther, B. (1988). Mode of peptidoglycan synthesis in Salmonella typhimurium: single strand insertion. J Bacteriol 170, 3509-3512.

Cynamon, M. H. \& Palmer, G. S. (1983). In vitro activity of amoxicillin in combination with clavulanic acid against $\mathrm{Myco-}$ bacterium tuberculosis. Antimicrob Agents Chemother 24, 429431.

Deng, L., Mikusova, K., Robuck, K. G., Scherman, M., Brennan, P. J. \& McNeil, M. R. (1995). Recognition of multiple effects of ethambutol on metabolism of mycobacterial cell envelope. Antimicrob Agents Chemother 39, 694-701.

Doyle, R. J., Chaloupka, J. \& Vinter, V. (1988). Turnover of cell walls in microorganisms. Microbiol Rev 52, 554-567.

Fischer, H. \& Tomasz, A. (1984). Production and release of peptidoglycan and wall teichoic acid polymers by pneumococci treated with $\beta$-lactam antibiotics. J Bacteriol 157, 507-513.

Giles, A. F. \& Reynolds, P. E. (1979). The direction of transpeptidation during cell wall peptidoglycan biosynthesis in Bacillus megaterium. FEBS Lett 101, 244-248.

Goodell, E. W. \& Schwarz, U. (1983). Cleavage and resynthesis of peptidoglycan cross-bridges in Escherichia coli murein. J Bacteriol 156, 136-140.

Hancock, I. C. (1981). The biosynthesis of ribitol teichoic acid by toluenised cells of Bacillus subtilis. Eur J Biochem 119, 85-90.

Hancock, I. C. \& Poxton, I. R. (1988). Bacterial Cell Surface Techniques, pp. 182-183. Chichester: Wiley.
Mauck, J. \& Glaser, L. (1972). On the mode of in vivo assembly of the cell wall of Bacillus subtilis. J Biol Chem 247, 1180-1127.

Merad, T., Archibald, A. R., Hancock, I. C., Harwood, C. R. \& Hobot, J. A. (1989). Cell wall assembly in Bacillus subtilis. J Gen Microbiol 135, 645-655.

Mikusova, K., Mikus, M., Besra, G. S., Hancock, I. C. \& Brennan, P. J. (1996). Biosynthesis of the linkage region of the mycobacterial cell wall. J Biol Chem 271, 7820-7828.

Mikusova, K., Yagi, T., Stern, R., McNeil, M. R., Besra, G. S., Crick, D. C. \& Brennan, P. J. (2000). Biosynthesis of the galactan component of the mycobacterial cell wall. J Biol Chem 275, 33890-33897.

Navarre, W. W. \& Schneewind, O. (1999). Surface proteins of Gram-positive bacteria and mechanisms of targeting to the cell envelope. Microbiol Mol Biol Rev 63, 174-232.

Pan, F., Jackson, M., Ma, Y. \& McNeil, M. (2001). Cell wall core galactofuran synthesis is essential for growth of mycobacteria. J Bacteriol 183, 3991-3998.

Periti, P. \& Mazzei, T. (1998). Antibiotic-induced release of bacterial wall components in the pathogenesis of sepsis and septic shock: a review. J. Chemother 10, 427-448.

Pooley, H. M. (1976). Turnover and spreading of old wall during surface growth of Bacillus subtilis. J Bacteriol 125, 1127-1138.

Takayama, K., Schnoes, H. K., Armstrong, L. \& Boyle, R. W. (1975). Site of inhibitory action of isoniazid in the synthesis of mycolic acids in Mycobacterium tuberculosis. J Lipid Res 16, 308-317.

Trevelyan, W. E., Proctor, D. P. \& Harrison, J. S. (1950). Detection of sugars on paper chromatograms. Nature 166, 444-445.

Ward, J. B. (1981). Teichoic and teichuronic acids: biosynthesis, assembly and location. Microbiol Rev 45, 211-243.

Ward, J. B. \& Perkins, H. (1974). Peptidoglycan synthesis by preparations from Bacillus licheniformis: cross-linking of newly synthesised chains to preformed cell wall. Biochem J 139, 781-784.

Waxman, D. J., Yu, W. \& Strominger, J. L. (1980). Linear uncrosslinked peptidoglycan secreted by penicillin-treated $B a$ cillus subtilis. J Biol Chem 255, 11577-11587.

Xin, Y., Lee, R. E., Scherman, M. S., Khoo, K.-H., Besra, G. S., Brennan, P. J. \& McNeil, M. (1997). Characterisation of the in vitro synthesised arabinan of mycobacterial cell walls. Biochim Biophys Acta 1335, 231-234.

Received 16 April 2002; revised 2 July 2002; accepted 5 July 2002. 